\title{
- Superconductivity in Bad Metals
}

\section{J. EMERY ${ }^{1}$ and S. A. KIVELSON ${ }^{2}$}

${ }^{1}$ Dept. of Physics, Brookhaven National Laboratory, Upton, NY 11973-5000

${ }^{2}$ Dept. of Physics, UCLA, Los Angeles, CA 90095

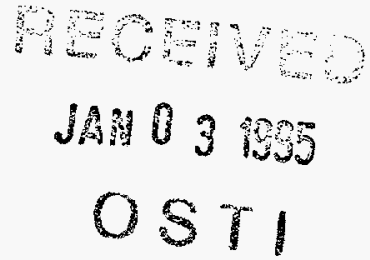

\begin{abstract}
It is argued that many synthetic metals, including high temperature superconductors are "bad matals", with such a poor conductivity that the usual mean-field theory of superconductivity breaks down because of anomalously large classical and quantum fluctuations of the phase of the superconducting order parameter. Some consequences for high temperature superconductors are described.
\end{abstract}

KEYWORDS: high- $T_{c}$, phase fluctuations, conductivity, superfluid density

\section{BAD METALS}

Synthetic metals, such as organic conductors, alkali-doped $C_{60}$, and high temperature superconductors, exhibit many of the phenomena of central interest in modern condensed matter physicssuperconductivity, metal-insulator transitions, and various charge or spin-ordered states. Typically these materials are strongly-correlated electron systems with a poor conductivity and a rather low effective carrier density, and it is natural to ask if we should view their properties, and in particular the mechanism of charge transport, in the way in elemental metals, such as copper or gold.

In the theory of simple metals it is often assumed that the quasiparticle decay rate and the transport scattering rate in a metal must be small compared to the quasiparticle energy $k_{B} T$, as required by Fermi liquid theory. However, quite ordinary metals, such as lead, fail to satisfy this condition at room temperature and yet their transport phenomena are well understood in terms of Boltzmann theory [1]. In fact, the concept of a propagating quasiparticle apparently does not break down entirely until its mean free path is shorter than its de Broglie wavelength: $l<\lambda_{F}=2 \pi / k_{F}$ [2]. In normal metals with strong electron-phonon coupling, a symptom of this breakdown is resistivity saturation; for the A15's, for example, the saturation value of the resistivity $(\rho \approx 150 \mu \Omega-c m)$ corresponds to $l \approx \lambda_{F}[1]$.

Typical synthetic metals are, in fact, "bad metals" in the sense that their resistance has a metallic (increasing) temperature dependence but, according to Boltzmann transport theory, the mean free path $l$ of a quasiparticle would be less than its de Broglie wavelength $\lambda_{F}$ at sufficiently high temperatures. This behavior is associated with the failure of bad metals to exhibit resistivity saturation. For example, in $\mathrm{La}_{1.85} \mathrm{Sr}_{.15} \mathrm{CuO}_{4-6}$, the resistivity in the a-b plane is a linearly increasing function of temperature from $T_{c}$ up to $900 \mathrm{~K}$, where its magnitude is about $0.7 \mathrm{~m} \Omega \mathrm{cm}$. According to Boltzmann transport theory this behavior implies $l / \lambda_{F}=0.4$ at $900 \mathrm{~K}$ with no sign of saturation. The failure of bad metals to exhibit resistivity saturation strongly suggests that any theory based on conventional quasiparticles with reasonably well-defined crystal momenta suffering occasional scattering events does not apply. Since the temperature dependence of the resistivity is unchanged as the temperature is lowered, this conclusion applies by continuity at lower temperatures, even though the estimated value of a mean free path would not, of itself, exclude quasiparticle transport.

This behavior has many important consequences. In particular, the anomalous behavior of the normal state of a bad metal requires that the physics of the superconducting transition be reconsidered. Here 
we give a summary of the main ideas and argue that the usual mean field theory breaks down because quantum and classical phase fluctuations may not be neglected. More detailed accounts are given elsewhere [3,4].

In the BCS-Eliashberg mean-field theory [5], which is an extremely good approximation for conventional superconductors, electron pairing and long-range phase coherence occur at the same temperature $T_{c}^{M F}$. There are essentially three types of fluctuations about the BCS ground state which can ultimately destroy the superconducting order: classical phase fluctuations, quantum phase fluctuations, and the effects of all other degrees of freedom which affect the local magnitude of the order parameter.

The stiffness of the system to classical phase fluctuations is determined by the superfluid density, $\rho_{s}(T)$; the smaller the superfluid density, the more significant classical phase fluctuations. In bad metals, especially the high temperature superconductors, the value of the bare superfluid density $\boldsymbol{n}_{s 0}$ is quite low at zero temperature, so the classical phase ordering temperature, which is proportional to $n_{s 0} / m^{*}$, can be substantially lower than $T_{c}^{M F}$.

Quantum phase fluctuations are associated with the number-phase uncertainty relation [6] according to which phase coherence between neighboring regions implies large relative number fluctuations and correspondingly large (oulomb energies unless there is adequate screening. Thus, the smaller the dielectric function. or equivalently the frequency dependent conductivity, the more significant the effects of quant um phase fluctuations.

Together, these efferts determine the superconducting transition temperature $T_{c}$ in a bad metal. But they have a number of other consequences for the physical properties of the materials. Local superconducting fluctuations are important for a much larger range of temperatures above $T_{c}$ than in good metals, and whase fluctuations control the temperature-dependence of the penetration depth and other paramelers of the superconducting state. In particular, the unusual linear temperaturedependence of the pentration depth of high temperature superconductors [7], may be explained in this way [3].

\section{CLASSICAL PHASE FLUCTUATIONS}

The import ance of phase fluctuations may be assessed by using experimentally-determined quantities to evaluate the temperature $T_{\theta}^{\max }$ at which phase order would disappear if the disordering effects of all other degrees of freedom were ignored. If $T_{c} \ll T_{\theta}^{\max }$, phase fluctuations are relatively unimportant, and $T_{c}$ will be close to the mean-field transition temperature, $T^{M F}$, predicted by BCS-Eliashberg theory [5]. On the other hand, if $T_{\theta}^{\max } \sim T_{c}$, then the value of $T_{c}$ is determined primarily by phase ordering, and $T^{M F}$ is simply the characteristic temperature below which local pairing becomes significant.

In order to evaluate $T_{\theta}^{m a x}$, the system must be divided into regions of linear dimension $a$ which are large enough for the order parameter to be well-defined locally. A region $j$ is characterised by a phase angle $\theta_{j}$ and its dynamically conjugate variable the number of electrons $N_{j}$. The Hamiltonian governing the thermodynamic effects of long wavelength phase fluctuations at low temperature is the kinetic energy of the superfluid:

$$
\mathcal{H}=\frac{1}{2} \rho_{s}(0) \int d \vec{r} \vec{v}_{s}^{2}
$$

where $\vec{v}_{s}=\hbar \nabla \theta / 2 m^{*}$ is the superfluid velocity, and $m^{*}$ is the effective mass of an electron. The system described by $\mathcal{H}$ undergoes a phase-ordering transition, since $\theta$ is an angle variable (defined modulo $2 \pi$ ), and there is a short-distance spatial cutoff, i.e. the variables are defined in regions of 
size $a$, and the integral and derivative in Eq. (1) should be regarded as a sum and a finite difference respectively.

The characteristic energy scale for phase fluctuations is the zero-temperature "phase stiffness" $V_{0}=$ $\left(\hbar / 2 m^{*}\right)^{2} \rho_{s}(0) a$, which may be expressed in terms of the length scale $a$ and a measurable quantity, the penetration depth $\lambda(T)$, via the relation $4 \pi \rho_{s}(0)=\left(m^{*} c / e \lambda(0)\right)^{2}$ to obtain:

$$
V_{0}=\frac{(\hbar c)^{2} a}{16 \pi e^{2} \lambda^{2}(0)}
$$

Since $V_{o}$ gives the energy scale of the model, it follows that the transition temperature $T_{\theta}^{\max }=A V_{0}$ where $A$ is a dimensionless number of order 1 which depends on the details of the short-distance physics. For three dimensions, our prescription for the short-distance cutoff is equivalent to defining the model on a simple cubic lattice, for which $A=2.2$ [8]. In order to complete the specification of $V_{0}$, we identify $a^{2}$ with the area $\pi \xi^{2}$ defined by the superconducting coherence length $\xi$; the precise numerical relation between $a$ and $\xi$ will not be important for the subsequent discussion.

Quasi two-dimensional systems, such as oxide superconductors consist of weakly coupled planes in which the phase variables may be defined on a square lattice. The value of $A$ lies between 0.9 (for a two-dimensional system [9]). and 2.2 (for the isotropic three-dimensional system). For definiteness, we shall use $A=0.9$ for all quasi-two dimensional materials, but the true phase ordering temperature may typically be $50 \%$ larger. The in-plane cutoff does not enter the expression for $V_{0}$, so that $a$ in Eq. (2) is the larger of the average spacing between layers $d$ or $\sqrt{\pi} \xi_{\perp}$, where $\xi_{\perp}$ is the coherence length perpendicular to the layers. Since $d$ is known very accurately, there is much less uncertainty in the calculated value of $V_{0}$ for materials, such as organic and high temperature superconductors, for which $d>\xi_{\perp}$.

Other ways of introducing the short-distance cutoff into the Hamiltonian will give somewhat different values of $A$ but the one we have chosen is physically natural and, as we shall see, it leads to a very suggestive interpretation of the phase diagram of high-temperature superconductors.

$T_{\theta}^{m a x}$ is an upper bound on the true superconducting transition temperature $T_{c}$ because of the neglect of quantum phase fluctuations, as well as the temperature dependent effects of the other degrees of freedom. Similarly, $T^{M F}$ is also an upper bound on $T_{c}$, since phase fluctuations will always depress $T_{c}$ somewhat. Thus, the ratio $T_{\theta}^{\max } / T_{c}$ provides a very useful criterion for the importance of phase fluctuations; if it is large, it is clear that phase fluctuations have a minor effect on $T_{c}$, whereas if it is close to 1 , then phase fluctuations depress $T_{c}$ substantially below $T^{M F}$ or, as we shall see, they may even be the major factor in determining $T_{c}$ itself. In fact $V_{0}$ is important in its own right since, as a measure of the rigidity of the superconducting state to variations of the phase, it characterises the energetics of vortex lattices.

The values of $T_{\theta}^{\max } / T_{\mathrm{c}}$ for a wide variety of materials are tabulated in reference [4]. For elemental metals such as lead, $T_{\theta}^{\text {max }} / T_{c} \sim 10^{5}$ is extraordinarily high; phase fluctuations have a negligible effect on $T_{c}$ and the superconducting state has a substantial phase rigidity at all temperatures below $T_{c}$. In other words, pairing and long-range phase coherence occur essentially simultaneously. With the exceptions to be described below, other superconducting materials behave in the same way, although the values of $T_{\theta}^{\max } / T_{c}$ are somewhat smaller.

On the other hand, the organic superconductor (BEDT-TTF) ${ }_{2} \mathrm{Cu}(\mathrm{NCS})_{2}$ and the hole-doped oxide superconductors are in an entirely different range of parameters inasmuch as $T_{\theta}^{\text {max }} / T_{c}$ is of order unity. In particular, the analysis suggests a new interpretation of the usual phenomenological classification of high temperature superconductors into three, more or less distinct categories: "underdoped", "optimally doped", and "overdoped." The value of $T_{c}$ is predominantly determined by phase fluctuations in underdoped high temperature superconductors such as $\mathrm{YBa}_{2} \mathrm{Cu}_{4} \mathrm{O}_{8}$, $\left(T_{\theta}^{m a x} / T_{c} \approx 1\right)$, and by the mean-field transition temperature $T^{M F}$ in overdoped materials such as 
Tl $2201\left(T_{\theta}^{\max } / T_{c} \geq 2\right)$. Optimally doped materials, such as $\mathrm{YBa}_{2} \mathrm{Cu}_{3} \mathrm{O}_{7-\delta}$ and $\mathrm{La}_{2-x} \mathrm{Sr}_{x} \mathrm{CuO}_{4}$, with $\delta$ and $x$ in the neighbourhood of 0.05 and 0.15 respectively, are in the crossover region between the two. Of course there is no precise dividing line between the two kinds of superconductor but rather a more or less gradual crossover in behavior as the value of $T_{\theta}^{\max } / T_{c}$ changes. Furthermore, the values of this ratio are subject to the uncertainties in the experimental values of $\lambda(0)$ and the precise form of the short-distance cutoff. But the systematic variation of the properties of high temperature superconductors from one material to another clearly supports the importance of phase fluctuations.

When $T_{c}$ is much smaller than $T^{M F}$, the effects of pairing manifest themselves as a pseudogap in the the temperature range $T_{c}<T<T^{M F}$. This observation provides a natural explanation of a variety of measurements, including NMR [10] and optical conductivity $[11,12]$, on underdoped high temperature superconductors, such as the stoichiometric material $\mathrm{YBa}_{2} \mathrm{Cu}_{4} \mathrm{O}_{8}\left(T_{c}=80 \mathrm{~K}\right)$, which show a pseudogap opening below a temperature of order $160-180 \mathrm{~K}$. Similar behavior is seen [10] in underdoped $\mathrm{YBa}_{2} \mathrm{Cu}_{3} \mathrm{O}_{7-\delta}$, extending to the large $\delta$ end of the the $90 \mathrm{~K}$ plateau, beyond which there is a rather rapid change in behavior as the oxygen content is increased.

These considerations are macroscopic and independent of the underlying "mechanism" of high temperature superconductivity. However, some additional constraints on the appropriate microscopic theory emerge: 1) The theory must account for the very high values of $T^{M F}>T_{c}$ and be able to survive any loss of low-energy spectral weight due to the opening of a pseudogap. 2) The fundamental reason why phase fluctuations are so important in the oxide superconductors is that they are doped insulators with a very low Drude weight which, in turn, implies a low superfluid density. This requires a significant departure from the BCS-Eliashberg theory. 3) When phase fluctuations are important, the characteristic consequences of the BCS mean-field theory, such as the existence of NMR coherence peaks, the jump in the specific heat at $T_{c}$, the value of the gap ratio, $2 \Delta / k_{B} T_{c}$, the isotope effect, and the temperature dependence of physical properties must be modified.

These ideas give a new perspective on the relation between $T_{c}$ and $\lambda^{-2}(0)$ suggested by muon spin relaxation experiments. From our point of view, the "universal relation" between $T_{c}$ and the muon depolarization rate in high temperature superconductors proposed by Uemura and coworkers [13] should in fact be reinterpreted as an upper bound on $T_{c}$ given by the ordering temperature for phase fluctuations, as shown below Eq. (2). Indeed the picture that underdoped materials are close to the bound (but depressed below it by an amount that depends on the conductivity) and that overdoped materials are further from the bound because the mean field transition temperature takes control, gives a very good description of the systematics of the relation between $T_{c}$ and the muon depolarisation rate.

\section{QUANTUM PHASE FLUCTUATIONS}

In elemental superconductors, $\sigma\left(T_{c}\right) \gg \sigma_{Q}$, so quantum phase fluctuations are entirely insignificant. However, as indicated above, poor conductivity implies poor screening, which supresses the charge fluctuations implied by phase order. A method of calculating these effects has been presented elsewhere [3]. Two consequences will be mentioned here.

In the case of pristine stoichiometric high temperature superconductors, $\sigma\left(T_{c}\right) / \sigma_{Q} \sim 10$, and the suppression of $T_{c}$ below $T_{\theta}^{m a x}$ is significant. According to our estimates, quantum fluctuations produce a $5 \%-7 \%$ suppression of $T_{c}$ in optimally-doped $\mathrm{YBa}_{2} \mathrm{Cu}_{3} \mathrm{O}_{7-\delta}$. Damaged or relatively more disordered samples tend to have a substantially smaller conductivity, and quantum effects are correspondingly more important. Indeed it is possible to increase the resistance so as to destroy superconductivity altogether. The idea that the superconducting transition is influenced by the value of the conductivity at $T_{c}$ correctly predicts many trends in the transition temperature of high 
temperature superconductors. It has been considered as an explanation of the variation of $T_{c}$ upon purposely reducing the conductivity of $\mathrm{YBa}_{2} \mathrm{Cu}_{3} \mathrm{O}_{7-\delta}$ by radiation damage [14].

A second important implication for high temperature superconductors is the existence of a substantial range of temperatures in the neighborhood of $T_{c}$ in which critical phase fluctuations dominate the low frequency electromagnetic response, and a low temperature regime in which $n_{s}(T)$ will have an anomalous temperature dependence as a result of phase fluctuations: $n_{s}(T) / n_{s}(0)=$ $1-\alpha \mathcal{F} k_{B} T /\left[2 V_{o}(\alpha-1)\right]$, to first order in $T$. Here, it is assumed that the Coulomb interaction is screened by the finite residual far IR conductivity observed in $\mathrm{YBa}_{2} \mathrm{Cu}_{3} \mathrm{O}_{7-\delta}$ [15]. If we use $\sigma(\omega)=500(\Omega \mathrm{cm})^{-1}$, (which is roughly consistent with experiment) together with $R_{Q} \approx 40 \mathrm{k} \Omega$ $(\mathcal{F} \approx 1 / 6)$, as deduced from the data of Fig. 1 , the linear temperature dependence of the superfluid density agrees quantitatively with the observations [7].

Acknowledgements: SK was supported in partby NSF grant \#DMR -90-11803. This work also was supported by the Division of Materials Sciences, U.S. Department of Energy, under contract DE-AC02-76CH00016.

[1] Allen PB Comments (1992) Cond. Mat. Phys. 15: 327-331

[2] Ioffe AF, Regel AR (1960) Semicond. 4: 237-251

[3] Emery VJ, Kivelson SA (1995) Phys. Rev. Lett. 74: 3253-3256

[4] Emery VJ, Kivelson SA (1995) Nature 374: 434-437

[5] Schrieffer JR (1964) Theory of Superconductivity. W. A. Benjamin. New York

[6] Anderson PW (1966) in Quantum Fluids, ed. Brewer DF North-Holland. Amsterdam, pp 146-171

[7] Hardy WN, Bonn DA, Morgan DC, Llang R, Zhang K (1993) Phys. Rev. Lett. 70: 3999-4003

[8] Adler J, Holm C, Janke W (1993) Physica A 201: 581-592

[9] Olsson P, Minnhagen P (1991) Physica Scripta 43: 203-209

[10] For a review see Mehring M (1992) Appl. Magn. Reson. 3: 383-421

[11] Basov DN, Timusk T, Dabrowski B, Jorgensen JD (1994) Phys. Rev. B 50: 3511-3514

[12] Wachter P, Bucher B, Pittini R (1994) Phys. Rev. B49, 13164-13171

[13] Uemura YJ (1989) Phys. Rev. Lett. 62: 2317-2320

[14] Sun AG, Paulius LM, Gajewski DA, Maple MB, Dynes RC (1994) Phys. Rev. B 50: 3266

[15] Basov DN, Liang R, Bonn DA, Hardy WN, Dabrowski B, Quijada M, Tanner DB, Rice JP, Ginsberg DM, Timusk T (1995) Phys. Rev. Lett. 74: 598-601

\section{DISCLAIMER}

This report was prepared as an account of work sponsored by an agency of the United States Government. Neither the United States Government nor any agency thereof, nor any of their employees, makes any warranty, express or implied, or assumes any legal liability or responsibility for the accuracy, completeness, or usefulness of any information, apparatus, product, or process disclosed, or represents that its use would not infringe privately owned rights. Reference herein to any specific commercial product, process, or service by trade name, trademark, manufacturer, or otherwise does not necessarily constitute or imply its endorsement, recommendation, or favoring by the United States Government or any agency thereof. The views and opinions of authors expressed herein do not necessarily state or reflect those of the United States Government or any agency thereof. 


\section{DISCLAMMER}

Portions of this document may be illegible in electronic image products. Images are produced from the best available original document. 